IDENTITE NATIONALE ET CONSTITUTIONNELLE DANS

LA JUDISPRUDENCE DE LA COUR DE JUSTICE DE L'UNION EUROPÉENNE'

\title{
NATIONAL AND CONSTITUTIONAL IDENTITY IN THE CASE LAW OF THE COURT OF JUSTICE OF THE EUROPEAN UNION
}

\author{
Ondrej Hamul'ák, David Kopal, Tanel Kerikmäe \\ Palacký University Olomouc, Faculté de droit, La Chaire Jean Monnet \\ Université de Technologie de Tallinn, École de commerce et de gouvernance, \\ département de droit Tallinn Law School
}

Résumé: Le but de cet article est de déterminer la position de la CJUE à l'égard de l'identité nationale en ce qui concerne sa jurisprudence et si la Cour privilégie l'identité nationale ou la primauté du droit de l'UE lors de l'équilibre entre les principes constitutionnels et Les intérêts des États membres avec le droit de l'UE. La partie introductive d'article traite de l'insertion et du développement de la clause d'identité nationale dans le droit primaire de l'UE. Sa partie principale consiste à analyser la jurisprudence de la CJUE, ainsi que les avis des Avocats Généraux dans la période antérieure et postérieure à l'adoption du Traité de Lisbonne.

Mots-clés: identité nationale, identité constitutionnelle, Cour de justice de l'Union européenne, relation entre le droit national et le droit de l'UE

\begin{abstract}
The aim of this paper is to determine the position of the CJEU towards the national identity with regard to its case law and whether the Court gives preference to the national identity or to the primacy of EU law during the balancing between the constitutional principles and the interests of member states with EU law. The introductory part of the paper addresses the insertion and the development of the national identity clause in the primary law. Its main part consists of analysis of the case law of the CJEU, as well as of the opinions of Advocates General, in the period before and after the adoption of the Treaty of Lisbon.
\end{abstract}

Keywords: national identity, constitutional identity, Court of Justice of the European Union, relationship between national law and EU law

\section{INTRODUCTION}

En général on peut dire que l'identité nationale, ou bien l'identité constitutionnelle des États membres de l'UE est liée aux élements essentielles de leur constitution en relation avec la commu-

1 Cet article a été écrit au nom des projets GAČR no. 17-22322S (Vliv Listiny základních práv EU na ústavní právo zemí Visegrádské čtyřky) and IGA no. PF_2015_017 (Evropská unie a finanční krize: Jak se změnil vztah mezi právním rádem EU a právními řády členských států?). 
nauté politique de tel ou tel état. ${ }^{2}$ Le trait caractéristique de ces élements constitutifs est le fait qu'ils sont donnés et ainsi protégés contre un éventuel changement. Dans le cas de la présente étude il s'agit d'une modification ayant son origine dans le droit européen qui s'accroit sans cesse et qui impiète de plus en plus dans les spécificités constitutionnelles des pays membres.

Bien que le respect de l'identité nationale des États membres fait partie du droit de l'UE depuis son commencement, ${ }^{3}$ ce n'est que le Traité de Maastricht de 1992 qui en a fait la partie explicite. Conformément à l'article $\mathrm{F}$ :

1. L'Union respecte l'identité nationale de ses États membres, dont le système politique est fondé sur les principes démocratiques.

2. L'Union respecte les droits fondamentaux, tels qu'ils sont garantis par la Convention européenne de sauvegarde des droits de l'homme et des libertés fondamentales, signée à Rome le 4 novembre 1950, et tels qu'ils résultent des traditions constitutionnelles communes aux États membres, en tant que principes généraux du droit communautaire.

Ce sont de probables changements importés dans le cadre juridique de l'UE par le Traité de Maastricht qui en ce moment représentent la raison de l'implémentation de la dite stipulation. Quoi qu'il s'agissait de la citoyenneté européenne, l'union économique et monétaire ou bien la monnaie unique, tous les changements avaient un impact incontestable sur la suveraineté des États membres. Ces derniers se sont mis d'accord pour inclure une stipulation de sécurité dans les Traités mêmes pour régler dans une certaine mesure la gamme des compétences européenne à l'aide des stipulations garantissants le respect de l'UE aux identités nationnales des États membres. ${ }^{4} \mathrm{La}$ judisprudence, au fur et à mesure croissante des instances intérieures suprêmes, y va de pair, et ces dernières ont essayé de définir les limites du droit de l'UE dans les ordres juridiques intérieurs. La conception de l'identité nationale dans les Traités avait un caractère plûtot politique, ce qui n'était pas le cas par exemple de la Cour constitutionnelle fédérale ${ }^{5}$ et l'impact de ces désions sur le rapport du droit de l'UE et du droit national. C'était causé aussi par le fait qu'à l'époque l'article F du Traité sur l'Union européenne (TUE) ne relevait pas de la jurisprudence de la Cour de justice de l'Union européenne (CJUE).

Il résulte de la présente stipulation que la conception de la protection de l'identité était dans sa version d'origine liée surtout aux principes de démocratie. Cependant, il faut prendre en considération le fait que le paragraphe suivant parle expressément aussi de la protection des droits fondamentaux émanant des traditions constitutionnelles des États membres.

Malgré le fait que le droit primaire exprimait expressément le respect à l'identité nationale des États membres, le contenu réel de cette locution verbale n'était pas clair. En plus, la situation a été rendue compliquée par le fait que la Cour de justice à l'époque n'avait pas la compétence d'interpréter le contenu de la présente stipulation, il n'y avait donc pas d'instance pouvant mettre des contours fondamentaux à cette nouvelle conception.

2 MARTÍ, José Luis. Two Different Ideas of Constitutional Identity: Identity of the Constitution v. Identity of the People. In: ARNAIZ, Alejandro Saiz, LLIVINA, Carina Alcoberro (ed). National Constitutional Identity and European Integration. Cambridge: Intersentia, 2013, p. 20.

3 Conclusion de l'avocat général Miguel Poiares Madura du 8 octobre 2008 dans l'affaire Michaniki, C - 213/07, ECLI: EU:C:2008:544.

4 ARNAIZ, Alejandro Saiz, LLIVINA, Carina Alcoberro. Why Constitutional Identity Suddenly Matters: A Tale of Brave States, a Mighty Union and the Decline of Sovereignty. In: ARNAIZ, Alejandro Saiz, LLIVINA, Carina Alcoberro (ed). National Constitutional Identity and European Integration. Cambridge: Intersentia, 2013, p. 6

5 Par exemple la décision de la Cour constitutionnelle fédérale du 12 octobre 1993, BverfGE 89, 155 Maastricht-Urteil. 
La disposition mentionée a été modifiée et renumérotée par l'adoption du Traité d'Amsterdam en 1997 qui a déplacé la protection de l'identité nationale dans le troisième paragraphe et a abrégé son texte de façon à ce que d'après l'ancien article 6 du TUE:

1. L'Union reconnaît les droits, les libertés et les principes énoncés dans la Charte des droits fondamentaux de l'Union européenne, les principes communes aux États membres.

2. L'Union respecte les droits fondamentaux garantis par la Convention européenne de sauvegarde des droits de l'Homme et des libertés fondamentales signée à Rome le 4 novembre 1950 tels qu'ils résultent des traditions constitutionnelles communes aux États membres en tant que principes généraux du droit communautaire.

3. L'Union reconnait l'identité nationale de ses États membres.

$\mathrm{Vu}$ que le respect à l'identité nationale et les textes du deuxième paragraphe restaient maintenus, cela semblait montrer qu'il s'agissait d'une modification formelle du présent article. En même temps le nouveau paragraphe premier a été inclu, définissant les valeurs principales et les principes communs aux États membres, les fondements de l'UE. L'identité nationale dans le paragraphe 3 peut être regardée comme l'élément faisant un contrepoind aux principes communs définis dans le paragraphe $1 .^{6}$

L'art. 6 gardait sa forme jusqu'à l'adoption du Traité de Lisbonne qui a non seulement assemblé de facto le deuxième et troisième paragraphe, mais a aussi élargi son contenu. ${ }^{7}$ Conformément à l'actuel article 4, paragraphe 2 TUE:

L'Union respecte l'égalité des États membres devant les traités ainsi que leur identité nationale, inhérente à leurs structures fondamentales politiques et constitutionnelles, y compris en ce qui concerne l'autonomie locale et régionale. Elle respecte les fonctions essentielles de l'État, notamment celles qui ont pour objet d'assurer son intégrité territoriale, de maintenir l'ordre public et de sauvegarder la sécurité nationale. En particulier, la sécurité nationale reste de la seule responsabilité de chaque État membre.

A première vue, il est évident que dans l'article mentionné il ne s'agisse pas de la définition exhaustive de l'identité nationale mais plûtot d'une simple liste des signes qui y sont liés. L'article allégué n'apporte pas de réponse à la question de savoir quel contenu et quels impacts réels peuvent avoir une telle conception sur les relations de l'ordre juridique de l'UE et les ordres juridiques nationaux des États membres. ${ }^{2}$

De l'art. 4 paragraphe 2 du TUE résulte que la notion de l'identité nationale entre autre peut comprendre le repect de l'Union européenne aux fondements constitutionnels des États membres, c'est-à-dire à leur identité constitutionnelle. Il en est clair aussi de la jurisprudence de la CJUE analysée ci-dessous parce que quand la Cour de justice, respectivement l'avocat général renvoie à l'art. 4 paragraphe $2 \mathrm{du}$ TUE ou précèdents, il mentionne souvent des principes résultant du droit constitutionnel des États membres. Donc, l'Union européenne a l'obligation de respecter non seulement l'identité de ses États membres mais en même temps leur identité constitutionnelle représentant un certain sous-ensemble de l'identité nationale.

De l'autre côté il faut regarder aussi la systèmatique de la disposition mentionnée où l'art. 4 du TUE mentionne dans les autres paragraphe en plus de la protection de l'identité nationale aussi le principe

6 CLAES, Monica. National Identity: Trump Card or Up for Negotiation?. In: ARNAIZ, Alejandro Saiz, LLIVINA, Carina Alcoberro (ed). National Constitutional Identity and European Integration. Cambridge: Intersentia, 2013, p. 118.

7 L'obligation de respecter l'identité nationale des états membres a été introduit même dans le Préambule au Convention des droits fondamentaux de l'UE qui après la ratification du Traité de Lisbonne a le même statut que le droit primaire. 
de la transmission des compétences et surtout le principe de la coopération loyale. En vertu du principe mentionné en dernier « Les États membres facilitent l'accomplissement par l'Union de sa mission et s'abstiennent de toute mesure susceptible de mettre en péril la réalisation des objectifs de l'Union. »

Il en résulte qu'il faut toujours équilibrer le respect à l'identité nationale des États membres avec d'autres principes découlant du droit primaire, surtout avec les principes de la primauté et de l'effet direct du droit de l'UE. ${ }^{8}$

Dans ce sens il faut souligner le fait que l'art. 4 paragraphe 2 du TUE dépend nouvellement de la compétence de révision de la Cour de justice puisque conformément à l'art. 19 paragraphe 1 du TUE la Cour de justice « assure le respect du droit dans l'interprétation et l'application des traités. »C'est pourquoi il est à la CJUE d'interpréter dans sa jurisprudence la conception de l'identité nationale ou bien constitutionnelle, et de définir la mesure dans la quelle la spécificité constitutionnelle d'un des États membres peut motiver une exception du droit de l'UE.

La question se pose si une nouvelle définition de l'identité nationale dans l'art. 4 paragraphe 2 du TUE présente une modification essentielle du droit primaire ou bien s'il s'agit d'un simple remaniement de ce qui contenait le droit primaire déjà depuis le Traité de Maastricht.

D'après certains auteurs la formulation dans l'art. 4 paragraphe 2 du TUE pourrait apporter un équilibre dans les relations entre la CJUE préférant le principe de la primauté absolue du droit de l'UE et les cours constitutionnelles nationales estimant les constitutions intérieures comme une barrière contre les effets expansifs du droit de l'UE. ${ }^{9}$ D' après certains parmi eux la conception de l'identité nationale pourrait fonctionner comme une exception du principe de la primauté du droit de l'UE pouvant être utilisée par des cours constitutionnelles intérieures. ${ }^{10} \mathrm{D}$ 'après une autre opinion le texte de l'art. 4 paragraphe 2 du TUE ne présente aucune modification matérielle car ils subsument l'actuelle protection des traditions constitutionnelles des États membres, auparavant contenue dans l'art. 6 paragraphe 2 du TUE sous la conception de l'identité nationale. ${ }^{11}$

Nous nous penchons du côté de l'avis mentionné en dernier car en comparant l'ancien art. 6 paragraphe 2 et 3 du TUE et l'actuel art. 4 paragraphe 2 du TUE il en résulte que le contenu du point de vue matériel reste sans modification. Pour cette raison, nous ne supposont pas que l'art. 4 paragraphe 2 du TUE dans le contexte du Traité de Lisbonne devrait être opéré comme une disposition permettant aux États membres de servir d'exception du principe de primauté du droit européen.

Pour répondre à la question si l'art. 4 paragraphe $2 \mathrm{du}$ TUE pourrait présenter une obligation positive pour l'UE et ses institutions, il faut mettre au clair ce qui est le contenu réel de la conception de l'identité nationale. La présente étude essaie de répondre à la question de savoir quelle est la position de la CJUE dans sa pratique décisionnelle concernant la question de l'identité nationale, et si, dans la compensation des principes constitutionnels et des intérêts des États membres avec le droit de l'UE, elle donne la priorité à l'identité nationale ou bien si elle impose plûtot la primauté du droit de l'UE même au détriment des intérêts légitimes des États membres.

8 GREWE, Constance. Methods of Identification of National Constitutional Identity. In: ARNAIZ, Alejandro Saiz, LLIVINA, Carina Alcoberro (ed). National Constitutional Identity and European Integration. Cambridge: Intersentia, 2013, p. 39.

9 BOGDANDY, Armin von, SCHILL, Stephan. Overcoming Absolute Primacy: Respect for National Identity under the Lisbon Tready. Common Law Market Review, 2011, an. 48, p. 1418.

10 KUMM, Matthias, FERRERES COMELLA, Victor. The Primacy Clause of the Constitutional Treaty and the Future of Constitutional Conflict in the European Union. International Journal of Constitutional Law, an. 3, n. 2-3, p. 473-492.

11 MARTINICO, Giuseppe. What Lies behind Article 4(2) TEU. In: ARNAIZ, Alejandro Saiz, LLIVINA, Carina Alcoberro (ed). National Constitutional Identity and European Integration. Cambridge: Intersentia, 2013, p. 94. 
Le présent chapître est divisé en trois parties. La première partie est consacrée à la jurisprudence de la Cour de justice concernant l'identité nationale de la période avant le Traité de Lisbonne. La partie suivant traite l'évolution de la jurisprudence après l'adoption du Traité de Lisbonne. Et la dernière partie résume l'approche de la Cour de justice à la conception de l'identité nationale en vertu de sa jurisprudence des deux périodes analysées.

\section{LA JURISPRUDENCE DE LA PÉRIODE D'AVANT LE TRAITÉ DE LISBONNE}

\subsection{La Cour de justice}

La Cour de justice a mentionné pour la première fois la notion d'identité nationale avant son établissement dans le droit primaire. La décision Groener ${ }^{12}$ concernait le règlement national irlandais conditionnant la nomination à un poste permanent à plein temps de professeur dans les institutions publiques d'enseignement professionnel à la preuve d'une connaissance suffisante de la langue irlandaise. D'après la Cour de justice le métier d'un enseignant revêt un rôle important dans la mise en place et le maintien de la politique interne, de plus, dans le cadre d'un enseignement, l'utilisation de l'irlandais est un moyen d'expression de son identité nationale et sa culture. C'est pourquoi le droit européen primaire d'après CJUE n'interdit pas d'adopter la politique dont l'objectif est la protection et le sauvegarde de la langue officielle d'un état membre. ${ }^{13}$

L'implémentation d'une telle politique ne peut pas empiéter d'après la CJUE dans la liberté fondamentale telle que la libre circulation des travailleurs. C'est pourquoi les mesures applicables ne doivent en aucun cas être disproportionnées par rapport au but poursuivi et les modalités de leur application ne doivent pas comporter de discriminations au détriment des ressortissants d'autres États membres. ${ }^{14} \mathrm{La}$ CJUE est parvenue à ce que la législation irlandaise soit conforme aux exigeances de la disposition sur la libre circulation des travailleurs à l'intérieur de la Communauté.

Vu que ladite décision est la seule dans laquelle la CJUE a mentionné la conception de l'identité nationale avant sont implémentation dans les Traités, il est à conclure que la Cour de justice jusqu'à là n’y prêtait pas attention.

Il est possible de trouver preuves à l'appui plus de mentions sur l'identité nationale, surtout dans les conclusions des avocats généraux après l'adoption du Traité de Maastricht, grâce auquel la protection de l'identité nationale fait inéluctablement partie des Traités. La Cour de justice même dans ce temps là (avant l'adoption du Traité de Lisbonne) n'a mentionné la protection de l'identité nationale que dans deux de ses décisions. Les deux, comme l'arrêt Groener, concernaient une soidisante identité culturele.

La première décision concernait la Commission des Communautés européennes contre GrandDuché de Luxembourg ${ }^{15}$ sur légalité des conditions internes de la nationalité luxembourgoise pour l'emploi d'un enseignant dans le système de l'enseignement public que prévoit la constitution

12 L'arrêt de la Cour de justice du 28 novembre 1989, Groener, C-379/87, ECLI:EU:C:1989:599.

13 Ibid., points 18, 19.

14 Ibid., point 19.

15 Arrêt de la Cour de justice du 2 juillet 1996, Commission des Communautés européennes contre Grand-Duché de Luxembourg, C-473/93, ECLI:EU:C:1996:263. 
luxembourgoise. La question est posée sur le fait de savoir si cette stipulation est conforme à la libre circulation des travailleurs. D'après le gouvernement luxembourgois la condition de la nationalité est liée à la transmission des valeurs traditionnelles et constitue donc, eu égard à la situation particulière démographique du pays, une condition motivée de la sauvegarde de l'identité nationale du Grand-Duché de Luxembourg. Celle-ci ne pourrait en effet être préservée si la majeure partie du corps enseignant était constitué de ressortissants d'autres États membres. ${ }^{16}$

D'après l'avocat général Léger toutes les matières enseignées ne reflètent pas forcément les valeurs traditionnelles du pays même si certaines peuvent sans doute refléter l'identité nationale. Permettre aux ressortissants des autres États membres d'enseigner dans les écoles ne représente pas selon l'avocat général une menace pour l'identité nationale parce que ces ressortissants doivent remplir d'autres conditions strictes prescrites par le droit interne pour l'exercice de cet emploi. ${ }^{17}$

La Cour de justice est arrivée à la conclusion que les dispositions mentionnées ne relèvent d'aucunes exceptions à la libre circulation des travailleurs fixées par le droit interne. ${ }^{18}$ D'après la CJUE, cette conclusion ne peut pas être influencée même pas par le fait, que conformément à l'ancien art. F paragraphe 1 du TUE 1, le mantien des identités nationales des États membres est un objectif légitime du droit de l'UE autorisant la restriction des libertés fondamentales. Dans ce cas là d'après la CJUE, l'intérêt du Luxembourg peut être assuré effectivement par des moyens moins limitants. La restriction en question était trouvée disproporticionnelle car la sauvegarde de l'identité nationale ne peut pas justifier l'exclusion des ressortissants des autres États membres de l'ensemble des emplois du secteur de l'enseignement. ${ }^{19}$

La Cour de justice a fait les mêmes démarches aussi dans la deuxième décision qui concernait le Grand-Duché de Luxembourg ${ }^{20}$ et dont l'objet était la condition légale de la nationalité luxembourgoise dans l'exercice des activités de notaire. La question soulevée était si cette condition n'est pas en contradiction avec le principe de la liberté d'établissement. Le gouvernement luxembourgois invoquait que l'objectif de la condition en cause vise à assurer le respect de l'histoire, de la culture, des traditions et de l'identité nationale luxembourgoise au sens de l'ancien art. 6 paragraphe 3 du TUE. ${ }^{21}$

L’avocat général Pedro Cruz Villalón, dans ses conslusions, répétait seulement l'argumentation du gouvernement, en reliant la condition de la nationalité dans l'exercice des activités du notaire avec la protection de l'identité constitutionnelle du Luxembourg. ${ }^{22}$

La CJUE se préoccupait surtout du fait s'il est possible d'inclure la disposition interne en cause dans l'exception des libertés fondamentales d'établissement découlant des Traités. Ce n'était que par la suite qu'il mentionnait que même si la sauvegarde de l'identité nationnale des États membres représente un but légitime respecté par l'ordre juridique de l'Union, «l'intérêt invoqué par le GrandDuché peut toutefois être utilement préservé par d'autres moyens que l'exclusion, à titre général, des

16 Ibid., point 32.

17 Conclusion de l'avocat général Léger du 5 mars 1996 dans l'affaire Commission contre Grand-Duché de Luxembourg, C-473/93, ECLI:EU:C:1996:80, points 134, 137, 139.

18 Commission contre Grand-Duché de Luxembourg, C-473/93, point 33.

19 Ibid., points 24, 35.

20 Arrêt de la Cour de justice du 24 mai 2011, Commission européenne contre le Grand-duché de Luxembourg, C-51/08, ECLI:EU:C:2011:336. Même si la décision a été prise après l'adoption du Traité de Lisbonne, la procédure avait démarré avant cette date.

21 Ibid., point 72.

22 Conclusion de l'avocat général Pedro Cruz Villalóna du 14 septembre 2010 dans l'affaire Commission européenne contre le Grand-Duché de Luxembourg, C-51/08, ECLI:EU:C:2010:525, point 141. 
ressortissants des autres États membres $» .^{23}$ Ansi, d'après la Cour de justice la condition de nationalité pour la profession de notaire ne relève pas de l'exception de la libre circulation découlant du droit primaire.

Comme dans l'arrêt Groener, aussi dans ces deux décisions mentionnées, la CJUE n’a fait références sur la protection de l'identité nationale que subsidiairement. Elle procède d'abord en vertu de sa jurisprudence établie concernant des exceptions des libertés du marché intérieur dont le centre est l'application du principe de proportionalité. Non seulement ces décisions n'ont pas expliqué ce que la Cour de justice entend sous la notion de l'identité nationale, mais cette notion n'a même pas été utilisée comme argument principale influençant considérablement le résultat de la procédure.

Une autre décision mentionnée ne concerne pas directement l'identité nationale mais du point de vue matériel il s'agit d'une affaire similaire. Dans la décision Omega ${ }^{24}$ la CJUE a pris en considération la spécificité constitutionnelle de l'État membre pour justifier l'exception du droit de l'UE, ce qui autrement ne serait pas possible.

La ville allemande Bonn a interdit par un arrêté les jeux laser dont l'objectif étaient la simulation d'actes d'homicides sur d'autres personnes pour divertir avec justification qu'une telle forme de divertissement est contraire à la dignité humaine protégée par la constitution allemande. La question de la juridiction introduisante l'affaire était si cette interdiction ne porte pas l'atteinte à la libre circulation des services dans le champ du droit de l'UE pour les prestateurs de ces jeux.

D’après la CJUE les droits fondamentaux font " partie intégrante des principes généraux du droit dont la Cour assure le respect et que, à cet effet, cette dernière s' inspire des traditions constitutionnelles communes aux États membres. ${ }^{25}$ La Cour de justice n'a explicitement pas fait référence à l'identité nationale mais sur les traditions constitutionnelles des États membres stipulées dans l'ancien art. 6, paragraphe $2 \mathrm{du}$ TUE.

D’après la Cour de justice « il n'est pas indispensable, à cet égard, que la mesure restrictive édictée par les autorités d'un État membre corresponde à une conception partagée par l'ensemble des États membres en ce qui concerne les modalités de protection du droit fondamental ou de l'intérêt légitime en cause. ${ }^{26}$ Quoique d'après la Cour de justice la dignité humaine est protégée dans le champ du droit de l'UE, l'État membre a une large liberté pour délimiter le contenu et la portée dudit principe en prenant en considération les spécificités nationales. La CJUE a aussi souligné que l'ordre public ne peut être invoqué qu'en cas de menace réelle et suffisamment grave, affectant un intérêt fondamental de la société. ${ }^{27}$

La CJUE est parvenue à constater que la restriction était dans ce cas là proportionnelle parce qu'elle limitait seulement l'interdiction des jeux laser qui ont pour objet de tirer sur des cibles humaines et l'arrêté litigieux n'est pas allé au-delà de ce qui est nécessaire pour atteindre l'objectif poursuivi par les autorités nationales compétentes. D’après la CJUE l'interdiction adoptée pour des motifs de protection de l'ordre public est conforme au droit de l'UE. ${ }^{28}$

Ainsi, la Cour de justice, dans ce cas là, a laissé à l'autorité interne le soin de déterminer qu'il était important d'appliquer une certaine spécificité constitutionnelle dans un contexte concret. C'est

\footnotetext{
Commission européenne contre le Grand-Duché de Luxembourg, C-51/08, point 124.

Arrêt de la Cour de justice du 14 octobre 2004, Omega, C-36/02, ECLI:EU:C:2004:614.

Ibid., point 33

Ibid., point 37

Ibid., point 30

Ibid., points 40, 41 .
} 
seulement ensuite que la CJUE seule fixerait la portée de ladite spécificité sur le droit de l'UE. ${ }^{29} \mathrm{La}$ CJUE dans une certaine mesure respectait le dialogue entre elle-même et les autorités internes au lieu de disposer de façon autoritaire.

Même si dans cette décision la CJUE ne partait pas de la sauvegarde de l'identité nationale explicitement et qu'elle a renvoyé aux traditions constitutionnelles des États membres, son approche était similaire aux décisions mentionnée ci-dessus. De nouveau, la Cour de justice est partie de sa jurisprudence constante concernant les dérogations du droit de l'UE qui trouvent leur origine dans le droit européen même. Par cette attitude elle garde également le contrôle absolu sur le résultat de la procédure. ${ }^{30}$ Ce qui est intéressant c'est que l'avocat général Poiares Maduro considére l'approche dans la décision Omega comme exemple du respect à l'identité nationale. ${ }^{31}$

\subsection{Conclusions des avocats généraux}

Les autres acteurs contribuant aux débats sur le sujet de l'identité nationale durant la période avant Lisbonne furent les avocats généraux avec leurs conclusions devant la CJUE. La Cour de justice n’a pas ramassé l'éponge jetée par les avocats généraux et n’a pas basculé du côté de l'argumentation avec l'identité nationale.

Dans la décision la Royaume d'Espagne contre Eurojust, l'Espagne a attaqué le régime linguistique utilisé dans le recrutement dans Eurojust et demandait l'annulation de la disposition demandant de remplir certains documents en anglais. L'avocat général Miguel Poiares Maduro, dans sa conclusion, a signalé le fait qu'en vertu de l'ancien art. 6 paragraphe 3 du TUE l'Union respecte l'identité nationale des États membres et le respect de la diversité linguistique est l'un de ses aspects principaux. Le principe du respect de la diversité linguistique est en plus explicitement énoncé même dans la Charte des droits fondamentaux de l'UE. ${ }^{32} \mathrm{La}$ Cour de justice dans sa décision ne se préocupait pas du tout de l'argument sur l'identité nationale parce qu'elle trouvait le recours irrecévable.

La décision Marrosu Vassallo concernait la législation italienne, dont la conséquence était la différence de traitement dans le cadre du secteur privé et public, dans le cas de transformation des contrats de travail à durée déterminée en contrats à durée indéterminée. Le gouvernement italien a justifié qu'il était nécessaire de percevoir ce traitement distinct en considération des exigences constitutionnelles, à savoir les conditions assurant l'impartialité et l'efficacité de l'administration. ${ }^{33}$

En réaction à cette argumentation l'avocat général Poiares Maduro a estimé qu'il était nécessaire de reconnaitre « aux autorités nationales, et notamment aux juridictions constitutionnelles, la responsabilité de définir la nature des spécificités nationales pouvant justifier une telle différence de traitement. Celles-ci sont, en effet, les mieux placées pour définir l'identité constitutionnelle des États membres que l'Union européenne s'est donnée pour mission de respecter. Il reste, toutefois,

29 BESSELINK, Leonard F. M., National and Constitutional Identity before and after Lisbon, Utrecht Law Review, 2010, an. 6, n. 3, p. 46.

30 ZBÍRAL, Robert. Concept de l'identité nationale en tant qu'un nouvel élément dans la relation du droit interne et de l'Union : Acquis théoriques et professionnels. Právník (Juriste), 2014, an. 153, n. 2, p. 123.

31 Conclusion de l'avocat général Miguel Poiares Maduro du 8 octobre 2008 dans l'affaire Michaniki, C - 213/07, ECLI: EU:C:2008:544, point 32

32 Conclusion de l'avocat général Miguel Poiares Maduro du 16 décembre 2004 dans l'affaire Royaume d'Espagne contre Eurojust, C-160/03, ECLI:EU:C:2004:817, points 24, 35.

33 Conclusion de l'avocat général Miguel Poiares Maduro du 20 septembre 2005 dans l'affaire Marrosu Vassallo, C - 53/04, ECLI:EU:C:2005:569, point 39 
que la Cour a pour devoir de vérifier que cette appréciation est conforme aux droits et aux objectifs fondamentaux dont elle assure le respect dans le cadre communautaire. $~^{34}$ En l'espèce, d'après l'avocat général la nécesssité de préserver la voie du concours comme voie particulière d'accès à l'emploi dans les administrations publiques peut être tenue pour un objectif légitime justifiant, dans ce secteur, l'exclusion de la transformation des contrats de travail à durée déterminée en contrats à durée indéterminée. Cette mesure doit être proportionnelle, c'est-à-dire que les moyens employés sont nécessaires et appropriés pour atteindre l'objectif légitime recherché. ${ }^{35}$

L'avocat général Poiares Maduro s'exprimait sur la notion de l'identité nationale aussi dans la conclusion sur l'affaire Michaniki, ${ }^{36}$ qui concernait la compatibilité de la législation nationale permettant l'exclusion d'un entrepreneur de la procédure d'attribution d'un marché public de travaux figurant dans le droit de l'UE. Cette régulation est le résultat de la disposition de la constitution nationale qui a pour but la défense de pluralisme des médias. La question était de savoir si la disposition de la constitution greque n'était pas en contradiction avec la directive n. 93/37/CEE sur la coordination des marchés publics des travaux.

D’après l'avocat général l'identité constitutionnelle fait partie de l'identité nationale prévue dans l'ancien art. 6 paragraphe 3 du TUE et le respect de l'identité constitutionnelle constitue pour l'Union européenne un devoir qui s'impose à elle depuis l'origine. ${ }^{37}$ La jurisprudence de la CJUE a déjà tiré les conséquences que l'État membre " peut, dans certains cas et sous le contrôle bien évidemment de la Cour, revendiquer la préservation de son identité nationale pour justifier une dérogation à l'application des libertés fondamentales de circulation. ${ }^{38}$

L'État membre peut d'abord l'invoquer explicitement lui même comme motif légitime et autonome de dérogation à la règles de la libre circulation ou seul l'État membre peut développer sa propre acception d'un intérêt légitime de nature à justifier une entrave à une libre circulation. Comme l'exemple dudit motif, l'avocat général a évoqué l'affaire mentionnée Omega dans laquelle l'État membre invoquait la protection de la dignité humaine prévue dans sa constitution de nature à motiver la restriction de la libre circulation des services.

Ensuite, l'avocat général est parvenu à constater qu'un État membre peut invoquer le respect de l'identité nationale pour justifier l'évaluation des mesures constitutionnelles. Le respect de l'identité constitutionnelle des États membres ne peut pas être compris comme une défense absolue à l'égard de toutes les règles constitutionnelles nationales. De même que le droit communautaire prend en compte l'identité constitutionnelle des États membres, de même le droit constitutionnel national doit s'adapter aux exigences de l'ordre juridique de l'UE. ${ }^{39} \mathrm{Il}$ faut toujours, d'après l'avocat général, confronter l'intérêt résultant de l'identité nationale de l'État membre aux exigences de l'ordre juridique de l'UE, c'est-à-dire appliquer le test de proportionnalité. La disposition de la constitution greque d'après l'avocat général dans le cas en espèce méconnait le principe de proportionnalité.

La Cour de justice est arrivée au même résultat quand elle a mentionné qu'il convennait « de reconnaitre aux États membres une certaine marge d'appréciation aux fins de l'adoption de mesures

34 Ibid., point 40.

35 Ibid., points $43,44$.

36 Conclusion de l'avocat général Miguel Poiares Maduro du 8 octobre 2008 dans l'affaire Michaniki, C - 213/07, ECLI: EU:C:2008:544.

37 Ibid., point 31.

38 Ibid., point 32.

39 Ibid. point 33. 
destinées à garantir les principes d'égalité de traitement des soumissionnaires et de transparence. ${ }^{40}$ D'après la CJUE c'est justement l'État membre qui peut le mieux, en prenant compte des facteurs internes, identifier les situations propices à l'apparition de comportements susceptibles d'entraîner l'inobservation de ces principes. La volonté de l'État membre de garantir la transparence de la procédure des marchés publics réjoint l’objectif d’intérêt général que constitue le maintien du pluralisme et de l'indépendance des médias d'information. ${ }^{41}$ La Cour de justice a trouvé la mesure greque concrète disproportionnelle. La Cour de justice a ainsi de nouveau escamoté l'argumentation par l'identité nationale, cette fois-ci avec la référence à l'intérêt général de l'État membre.

Dans l'affaire Umweltanwalt von Kärnten ${ }^{42}$ l'avocat général a aussi essayé d'argumenter par la conception de l'identité nationale en mentionnant que les États membres de l'UE ont une compétence de souverainté quant à la définition de la composition et de la répartition des pouvoirs voulue par leur Constitution. Si un pays membre attribue des fonctions juridictionnelles à des organes parajudiciaires, il s'agit d'une volonté liée à l'identité constitutionnelle et à la souveraineté. ${ }^{43} \mathrm{D}^{\prime}$ après l'avocat général, le droit d'un État membre de décider la composition du pouvoir judiciaire national fait partie de l'identité constitutionnelle. La conclusion de l'avocat général n'a pas encore convaincu la Cour de justice de s'occuper de l'identité nationale.

La notion de l'identité nationale a été mentionnée dans d'autres conclusions des avocats généraux, par ex. en l'espèce de la protection d'une collectivité territoriale ${ }^{44}$ ou bien liée à la détermination des conditions de l'acquisition de la nationalité. ${ }^{45}$

L'effort de préciser l'interprétation de l'actuel art. 4 paragraphe 2 du TUE et de proposer à la Cour de justice dans ce sens des arguments pertinents est mis en lumière par toutes les conclusions mentionnées par des avocats généraux. C’est Poiares Maduro, sans doute, qui a le plus de mérite et d'après lui ce sont justement les autorités nationales des États membres qui définissent la structure de l'identité nationale. D'après lui, ce n'est qu'après que la Cour de justice doit estimer la proportionnalité de cette structure avec le droit de l'UE par l'intermédiaire du principe de proportionnalité. D’après l'avocat général l'argument de l'identité nationale peut directement justifier la dérogation au droit de l'UE. Cependant, sa référence à l'affaire Omega se montre un peu problématique, parce que comme il l'a été dit ci-dessus, dans cette décision le trait principal de l'argumentation de la Cour de justice reflétait sa jurisprudence constante, l'argumentaion des spécificités constitutionnnelles des États membres n'était utilisée que subsidiairement.

En résumé, dans sa période avant Lisbonne, en prenant en considération le nombre des décisions pertinentes, la CJUE dans la conception de l'identité nationale ne considére pas un argument qui pourrait être décisif lui même dans le cas où il s'agit d'une éventuelle atteinte aux spécificités constitutionnelles des États membres. Cette notion reste ainsi pour le moment « séquestrée » particulièrement dans les conclusions des avocats généraux.

\footnotetext{
40 Arrêt de la Cour de justice du 16 décembre 2008, Michaniki, C - 213/07, ECLI:EU:C:2008:731, point 55.

41 Ibid. points 55, 59.

42 Conclusion de l'avocat général Dámas Ruiz-Jarabo Colomer du 25 juin 2009 dans l'affaire Umweltanwalt von Kärnten, C-205/08, ECLI:EU:C:2009:397.

43 Ibid. point 47

44 Conclustion de l'avocat général Verici Trstenjak du 4 juin 2008 dans l'affaire Coditel Brabant, C-324/07, ECLI:EU: C:2008:317.

45 Conclusion de l'avocat général Miguel Poiares Maduro du 30 septembre 2009 dans l'affiare Rottmann, C-135/08, ECLI: EU:C:2009:588.
} 


\section{JURISPRUDENCE APRES LISBONNE}

\subsection{Le droit primaire}

Après l'adoption du Traité de Lisbonne, c'est-à-dire quand l'art. 4 paragraphe $2 \mathrm{du}$ TUE est devenu l'objet de la révision par la Cour de justice, la CJUE s'est exprimée concernant la notion de l'identité nationale pour la première fois dans l'affaire Sayn-Wittgenstein. ${ }^{46}$ Cette décision concernait la compatibilité de l'art. 21 TFUE régissant la liberté de circulation en application des règles autrichiennes prohibant les titres de noblesse. Ces dernières émanant du principe autrichien constitutionnel de l'égalité de traitemement. La requérante dans la procédure devant la cour nationale a affirmé que l'impossibilité de porter le titre de noblesse est une atteinte au droit de tout citoyen de l'Union de circuler librement. ${ }^{47}$ De l'autre côté, d'après le gouvernement autrichien, l'interdiction mentionnée constitue la sauvegarde de l'identité constitutionnelle de la République d'Autriche. ${ }^{48}$

D’après la CJUE le fait que les autorités de l'État membre aient refusé de reconnaitre tous ses élements du nom patronymique d'un ressortissant d'un État membre tel qu'il a été déterminé dans un second État membre constitue une restriction aux libertés reconnues dans l'art. 21 du TFUE à tout citoyen de l'UE. ${ }^{49}$ Ensuite, la Cour de justice a rappelé que « conformément à l'article 4, paragraphe 2, TUE, l'Union respecte l'identité nationale de ses États membres, dont fait aussi partie la forme républicaine de l'État. ${ }^{50}$ La loi autrichienne sur l'abolition de la noblesse, d'après la CJUE fait partie de l'histoire constitutionnelle autrichienne et constitue un élement de l'identité nationale qui peut être pris en compte lors de la mise en balance d'intérêts légitimes avec le droit de la libre circulation. Quoique l'identité nationale puisse être prise en compte, la justification du gouvernement autrichien invocant l'atteinte à l'identité constitutionnelle peut être malgré cela, d'après la CJUE, interprété comme quoi le gouvernement autrichien invoque l'ordre publique. ${ }^{51}$

D'après la CJUE cette notion doit être comprise de la façon restrictive et donc sa structure ne peut pas être déterminé unilatéralement par chaque État membre sans contrôle des autorités européennes. L'ordre public ne peut être invoqué « qu'en cas de menace réelle et suffisamment grave, affectant un intérêt fondamental de la société. ${ }^{52} \mathrm{Vu}$ que les circonstances pouvant justifier à avoir recours à la notion de l'ordre public peuvent être différentes dans chaque État membre, il faut reconnaître aux autorités nationales une marge d'appréciation. Avec la référence sur l'arrêt Omega, la CJUE a rappelé que la nécessité et la proportionnalité des mesures nationales ne sont pas exclues pour la seule raison que l'État membre a choisi le système de la sauvegarde qui est différent de celui offert par un autre état. ${ }^{53}$

Enfin, la CJUE est ensuite parvenue à l'opinion que l'art. 21 TFUE doit être interprété en ce sens qu'il ne s'opposait pas à ce que les autorités nationales, dans des conditions déterminées, refusaient

Arrêt de la Cour de justice du 22 décembre 2010, Ilonka Sayn-Wittgenstein, C-208/09, ECLI:EU:C:2010:806.

Ibid., point 43.

48 Ibid., point 74.

49 Ibid., point 71.

50 Ibid., point 92.

51 Ibid., points 83,84 .

52 Ibid., point 86

53 Ibid., points 87,91 
de reconnaitre, dans tous ses élements, le nom patronymique d'un citoyen de son pays. La restriction autrichienne de la liberté de circulation est dans ce cas là proportionnelle.

Même si la Cour de justice dans cet arrêt s'occupait de la sauvegarde de l'identité constitutionnelle, fondamentallement, elle a interprété la revendication du droit constitutionnel autrichien comme faisant partie de l'ordre public autrichien permettant une restriction nécessaire et proportionnelle des libertés fondamentales. ${ }^{54}$ L'art. 4 paragraphe 2 du TUE ne jouait pas un rôle important dans cette décision et il a été utlisé comme un argument subsidiaire, qui probablement n’influencait pas de point de vu considérable sur le résultat même.

Malgré ces faits, la CJUE a au moins tiré au clair la conception obscure d'interpréation de l'identité nationale parce qu'elle a attiré l'attention sur la relation solide entre l'identité nationale et les principes nationales constitutionnelles. Elle l'a fait non seulement par le fait qu'elle a relié l'identité nationale à l'intérêt résultant de la constitution autrichienne mais, en outre par le fait que d'après la CJUE la notion de l'État en tant que république fait partie de l'identité nationale. ${ }^{55}$ Le respect à la Constitution d'un État membre résulte aussi du fait qu' un éventuel conflit entre le droit constitutionnel et le droit de l'UE doit être régulé par le principe de proportionnalité.

La Cour de justice traite la conception de l'identité nationale aussi dans l'arrêt Runevič-Vardyn ${ }^{56}$ qui concernait la situation où les autorités lituaniennes ont refusé de modifier les noms et prénoms des requérants tels qu'ils figurent sur leurs actes d'état civil. Ces données, conformément aux lois lituaniennes, doivent être rédigées uniquement en caractères de l'aphabet latin, aucun autre caractère n'est autorisé. La question était la conformité de ladite législation avec la libre circulation des personnes en vertu de l'art. 21 du TFUE.

L'avocat générale Jääskinen a relevé dans sa conclusion que l'UE, conformément à l'art. 4 paragraphe 2 du TUE, honore l'identité nationale des États membres et donc, leur offre la possibilité de créer des règles de graphie des prénoms et des noms de famille tendant à faire respecter la langue nationale. ${ }^{57}$

La Cour de justice a fait d'abord référence à l'argumentation utilisée dans l'arrêt Groener, d'après laquelle le droit primaire européen n'interdit pas d'accepter la politique ayant pour but la sauvegarde et la promotion de la langue officielle d' un État membre. Ensuite, elle a poursuivi que tant aux termes de l'art. 3 paragraphe 3 du TUE ainsi que conformément à la Charte des droits fondamentaux de l'UE, l'Union respecte la diversité culturelle, religieuse et linguistique. Et ce n'était que subsidiairement qu'elle ajoutait que l'UE « respecte également l'identité nationale de ses États membres, dont fait aussi partie la protection de la langue officielle nationale de l'État. " ${ }^{58}$

Ensuite, elle relate que c'est à la juridiction nationale de faire valoir le principe de proportionnalité et de déterminer ainsi la conformité de la législation nationale avec la libre circulation des personnes en vertu de l'art. 21 du TFUE. ${ }^{59}$ D'après la CJUE la législation nationale qui vise à protéger la langue officielle nationale constitue un objectif légitime susceptible de justifier des restrictions aux droits de la libre circulation prévus dans l'article 21 du TFUE. ${ }^{60}$

En accord BOGDANDY: Overcoming Absolute Primacy Bogdandy ..., p. 1424

55 Ibid., p. 1425.

56 Arrêt de la Cour de justice du 12 mai 2011, Runevič-Vardyn, C-391/09, ECLI:EU:C:2011:291.

57 Conclusion de l'avocat général Niila Jääskinena du 16 décembre 2010 dans l'affaire Runevič-Vardyn, C-391/09, ECLI: EU:C:2010:784, point 80.

58 Runevič-Vardyn, C-391/09, points 85, 86.

59 Ibid., point 83.

60 Ibid., point 87. 
La CJUE a ensuite relevé que dans le cas où il a été démontré que le refus de modification du nom de famille commun pour un couple de citoyens de l'UE provoquait de sérieux inconvénients, il appartient de nouveau à la juridiction nationale « de déterminer si un tel refus respecte un juste équilibre entre les intérêts en présence, à savoir, d'une part, le droit des requérants au principal au respect de leur vie privée et familiale ainsi que, d'autre part, la protection légitime par l'État membre concerné de sa langue officielle nationale et de ses traditions. " ${ }^{61}$ Aussi, dans ce cas là, la Cour de justice a laissé à la juridiction nationale le soin de faire valoir le principe de proportionnalité.

Comme dans l'arrêt Sayn-Wittgenstein, l'art. 4 paragraphe 2 du TUE n'était pas appliqué individuellement comme un objetif légitime justifiant la dérogation au droit de l'UE mais comme un argument supplémentaire à côté de l'allégation principale, expliquant que la diversité linguistique résultait de l'art. 3 paragraphe 3 du TUE ainsi que de l'art. 22 de la Charte des droits fondamentaux de l'UE. De l'autre côté il faut mentionner que dans sa conclusion à la décision postérieure, l'avocat général Kokott a fait référence à l'interconnetion desdites stipulations en estimant que le fondement de l'art. 22 de la Charte des droits fondamentaux de l'UE reposait, d'après les explications officielles, sur le respect à l'identité nationale des États membres. ${ }^{62}$

Dans les deux cas suivants la CJUE n'a pas accepté l'argumentation par l'identité nationale pouvant justifier la dérogation au droit de l'UE. La première décision Anton Las ${ }^{63}$ concernait la question de la sauvegarde de la langue officielle. L'objet de la présente affaire était un décret belge adopté sur le fondement constitutionnelle qui exige que tout employé installé dans la région de langue néerlandaise ait des contrats de travail tranfrontaliers rédigés en néerlandais sous peine de nullité. La question était si cette législation, ayant une portée restreignante sur les employés ne parlant pas néerlandais et sur les employeurs des autres États membres, est en contradiction avec l'art. 45 du TFUE réglant la libre circulation des travailleurs.

D’après l'avocat général Jääskinen l'identité nationale comprend les aspects linguistiques de l'ordre constitutionnel d'un État membre qui définissent notamment la langue officielle, le cas échéant, les subdivisions territoriales dans lesquelles ces langues sont en usage. ${ }^{64}$ D'après Jääskinen l'identité nationale belge comprend la division de source constitutionnelle en communautés linguistiques et c'est pourqoui la politique de défense d'une langue est un motif pour autoriser un État membre à recourir à des mesures restreignant les libertés de circulations. ${ }^{65}$ Néanmoins, l'usage obligatoire de la langue résultant de la réglémentation en cause d'après l'avocat général, ne répond pas à cet objectif, puisque la rédaction des contrats transfrontaliers de travail dans une langue autre que le néerlandais n'est pas susceptible à menacer son usage. ${ }^{66}$

La Cour de justice, d'abord avec référence sur l'arrêt Runevič-Vardyn a invoqué qu'en vertu de l'art. 4 paragraphe 2 du TUE la défense de la langue officielle d'un État membre fait partie de l'identité nationale. ${ }^{67}$ L'objectif reposant sur le soutien de l'emploi de langue néerlandaise, qui est une des langues officielles du Royaume de Belgique, constitue d'après la CJUE un intérêt légitime de nature à

61 Ibid., point 91.

62 Conclusion de l'avocat général Juliane Kokott du 21 juin 2011 dans l'affaire République italienne contre Commission européenne, C 566/10 P, ECLI:EU:C:2012:368, point 86.

63 Arrêt de la Cour de justice du 16 avril 2013, Anton Las, C-202/11, ECLI:EU:C:2013:239.

64 Conclution de l'avocat général Niilo Jääskinen du 12 juillet 2012 dans l'affaire Anton Las, C-202/11, ECLI:EU:C:2012: 456 , point 59.

65 Ibid., point 60

66 Ibid., point 61

67 Anton Las, C-202/11, point 25. 
justifier la restriction aux obligations prévues dans l'art. 45 du TFUE. Ensuite, la Cour de justice est parvenue à ce que la législation belge dans cette affaire là aille au-delà de ce qui est strictement nécessaire pour atteindre l'objectif mentionné et ne saurait donc être considérée comme proportionnée. ${ }^{68}$

La Cour de justice s'est prononcée sur de l'identité nationale aussi dans l'affaire Digibet Albers ${ }^{69}$, concernant la réglementation des jeux de hasard. Plus exactement, la question était de savoir si l'existence de la réglementation juridique plus libérale des jeux de hasard, au moyen d'Internet, dans un des Länder, par rapport à la réglementation plus stricte dans d'autres Länders, représente une restriction illicite à la libre prestation des services en vertu de l'art. 56 du TFUE.

La CJUE jugait si une telle restriction peut être admise au titre des mesures dérogatoires aux l'art. 21 et 52 du TFUE pour des raisons d'ordre public, de sécurité publique et de santé publique ou bien conformément à sa jurisprudence motivée par des raisons impérieuses d'intérêt général. ${ }^{70}$

La Cour de justice, faisant référence à sa jurisprudence constante, alléguait que les autorités nationales bénéficiaient, dans le domaine des jeux de hasard, d'un large pouvoir d'appréciation pour déterminer les exigences pour la protection du consommateur et de l'ordre social. Si les conditions établies par la jurisprudence de la CJUE sont respectées, il appartient à chaque État membre d'apprécier si dans le contexte des buts légitimes qu' il poursuit, il est nécessaire d'interdire ou de restreindre des activités de hasard. ${ }^{71} \mathrm{La}$ CJUE s'est référée à l'identité nationale et a estimé que la répartition des compétences entre les Länder ne saurait être remise en cause puisqu'elle est protégée par l'art. 4 paragraphe 2 du TUE. ${ }^{72}$

D'après la Cour de justice l'art. 56 du TFUE doit être ainsi interprété dans le sens qu'il n'oppose pas la législation mentionnée des jeux de hasard plus libérale dans un Länder que dans les auters Länder. Mais cela n'est valable que si une telle législation est proportionnelle.

Dans cet arrêt la CJUE a pris l'argumentation par l'identité nationale de nouveau et seulement de façon indirecte parce qu'elle traitait surtout l'existence de la dérogation du droit primaire et non la problématique de l'organisation fédérale de l'Allemangne et dans le contexte de laquelle elle a mentionné l'identité nationale.

Comme dans l'arrêt Anton Las, aussi dans Digibet Albers la Cour de justice n'utilise de nouveau l'art. 4 paragraphe 2 TUE que subsidiairement pour soutenir le résultat auquel elle est parvenue par les démarches résultant de sa jurisprudence constante. Cette dernière lui donne une certaine liberté pour la réflexion de l'État membre.

En ce qui concerne le contenu de l'art. 4 paragraphe 2 du TUE, des quatre arrêts analysés résulte que cet article inclu les interêts qui concernent la prohibition des titres de noblesse, le respect au multilinguisme et l'organisation fédérale des États membres. L’arrêt Sayn-Wittgenstein, RunevičVardyn a Anton Las montrent d'un côté que les États membres peuvent recourir à l'art. 4 paragraphe 2 du TUE comme à la dérogation au droit primaire. Cependant, la CJUE n’a utilisé, dans aucun des cas, cette stipulation séparement, mais toujours liée à sa jurisprudence constante prévoyant les dérogations au droit de l'UE ou en commun au droit fondamental résultant de la Charte des droits fondamentaux de l'UE.

68 Ibid., points 27, 33.

69 Arrêt de la Cour de justice du 12 juin 2014, Digibet Albers, C-156/13, ECLI:EU:C:2014:1756.

70 Ibid., point 22.

71 Ibid., point 32

72 Ibid., point 34 . 


\subsection{Le droit secondaire}

La CJUE dans la période d'après le Traité de Lisbonne s'occupait de la question de l'identité nationale même dans les cas où les obligations des États membres résultaient du droit secondaire de l'UE. L'un des premiers arrêts en était l'affaitre O'Bien, ${ }^{73}$ ayant pour objet la question si les juges peuvent être compris dans l'accord-cadre sur le travail à temps partiel qui figure dans l'annexe de la directive 97/81/CE. D'après le gouvernement letton l'application du droit européen sur la juridiction d'un État membre impliquerait le non-respect de l'identité nationale des États membres en vertu de l'art. 4 paragraphe 2 du TUE. ${ }^{74}$

Mais la Cour de justice a refusé cet argument parce que d'après elle il appartient aux États membres de définir la notion de " travailleurs » en vertu de la directive et de l'accord-cadre pour déterminer si les juges relèvent de cette notion, à condition que cela n'aboutisse pas à exclure arbitrairement cette catégorie des personnes au bénéfice de la protection offerte par la directive et l'accord-cadre. L'exclusion d'un bénéfice de cette protection, d'après la CJUE, ne peut être admise que dans le cas où la relation entre les juges et le Ministère de la Justice est, de par sa nature, substantiellement différente de celle qui lie les employeurs et les employés. ${ }^{75}$

La CJUE a mentionné ensuite qu'appliquer la directive et l'accord-cadre sur les juges à temps partiel ne peut pas avoir d'effet sur l'identité nationale parce que l'objectif des deux documents n'était qu'à l'égard des juges, «à leur faire bénéficier du principe général d'égalité de traitement, qui constitue l'un des objectifs de ces textes, et ainsi à les protéger contre les discrimininations à l'égard des travailleurs à temps partiel. ${ }^{76}$ Ainsi, d'après la Cour de justice, en l'espèce, l'argumentation par l'identité nationale n'était pas pertinente.

Un autre cas concernant le droit secondaire était l'arrêt Commission européenne contre Royaume d'Espagne. ${ }^{77}$ Dans cette affaire on a opposé que l'Espagne a incorrectement transposé la directive $\mathrm{n}$. 200/60/CE, déteminant le cadre pour l'activité de la Communauté dans le domaine de la politique de l'eau, dans le droit national. Le gouvernement espagnol a invoqué que s'il s'agissait d'un bassin hydrographique intracommunautaire, la mise en oeuvre des obligations résultant de la directive était assurée par l'art. 149 paragraphe 3 de la Constitution espagnole stipulant que si la communauté autonome dispose dans un certain domaine du pouvoir législatif, et si cette dernière ne profite pas de ce pouvoir, pour les matières qui ne sont pas attribuées aux communautés autonomes, les normes d'état seront appliquées.

Mais d'après l'avocat général la non acceptation par l'UE de la conception de l'art. 149 paragraphe 3 de la Constitution espagnole ne contestait pas l'identité nationale de l'Espagne. « Il n'est pas exclu d'assurer la transposition du droit de l'Union, dans des États fédéraux ou décentralisés, par l'application subsidiaire des instruments de l'État central. Il faut simplement que cette application subsidiaire ne fasse aucun doute. Le droit espagnol ne satisfait pas à ces exigences. ${ }^{78}$

Arrêt de la Cour de justice du 1 mars 2012, O’Brien, C-393/10, ECLI:EU:C:2012:110.

74 Ibid., point 49

75 Ibid., point 51.

76 Ibid., point 49.

77 Arrêt de la Cour de justice du 24 octobre 2013, Commission européenne contre Royaume d'Espagne, C-151/12, ECLI: EU:C:2013:690.

78 Conclusion de l'avocat général Juliane Kokott du 30 mai 2013 dans l'affiare Commission européenne contre Royaume d'Espagne, C-151/12, ECLI:EU:C:2013:354, point 34. 
La Cour de justice approuvait la conclusion de l'avocat général et a mentionné que l'Espagne n'a pas rempli les conditions résultant de la directive. D’après la CJUE l'existence des principes généraux du droit constitutionnel et administratif peut rendre superflue la transposition par des mesures spécifiques à condition que ces principes garantissent la pleine application de la directive par l'administration nationale. ${ }^{79}$ Ensuite, la Cour de justice, concernant l'argument du Royaume d'Espagne selon lequel la Commission européenne aurait tenté en violation de l'art. 4 paragraphe 2 du du TUE déterminer la manière de la transposition des dispositions en cause, a constaté que la Commission européenne n’avait pas mentionné dans la requête la manière de la transposition des dispositions en cause de la directive. ${ }^{80}$ Ainsi, d'après la CJUE les spécificités constitutionnelles de l'Espagne ne sont pas pertinentes puisque cet argument repose sur l'accusation mal comprise que la Commission européenne a déposé contre l'Espagne.

La Cour de justice a adopté une approche similaire aussi dans l'arrêt Torresi ${ }^{81}$ Dans cette affaire la Cour de justice nationale s'est adressée à la CJUE avec la demande que, si l'art. 3 de la directive n. 98/5/CE permettant aux ressortissant italiens qui ont obtenu le titre professionnel d'avocat dans un État membre autre que la République italienne, le fait d'exercer leur activité dans cet état, fait contourner la constitution italienne qui subordonne l'accès à la profession d'avocat à l'obtention d'un examen d'État réussis. Donc, la question était si la disposition de la directive était en contradiction avec l'art. 4 du paragraphe 2 du TUE et du fait si elle devrait être considérée comme nulle.

D'après l'avocat général, l'adhésion au barreau des ressortissants de l'UE qui ont obtenu un titre professionnel dans un autre État membre, ne présenterai pas une menace pour l'identité nationale. ${ }^{82}$ Quoique la jurisprudence de la CJUE admet la possiblité que les États membres dérogent à leurs obligations résultant du droit européen pour des motifs de la protection de l'identité nationale, cela ne signifie pas « que toute règle consacrée dans une Constitution nationale puisse limiter l'application uniforme des dispositions de l'Union ${ }^{83}$.

La CJUE a renoué avec cette argumentation quand elle a estimé que l'art. 3 de la directive ne concerne que le droit de s'établir dans un État membre pour y exercer la profession d'avocat sous le titre professionnel délivré dans l'État membre d'origine. Cette disposition ne réglemente pas ainsi, d'après la CJUE, l'accès à la profession d'avocat ni l'exercice de cette profession sous le titre professionnel obtenu dans l'État membre d'accueil. ${ }^{84}$ " Il en résulte nécessairement qu' une demande d'inscription au tableau des avocats établis, présentée au titre de l'article 3 de la directive 98/5, n'est pas de nature à permettre d'éluder l'application de la législation de l'État membre d'accueil relative à l'accès à la profession d' avocat. ${ }^{85}$ L'art. 3 de la directive «n'est pas, en tout état de cause, susceptible d'affecter les structures fondamentales politiques et constitutionnelles ni les fonctions essentielles de l'État membre d'accueil au sens de l'article 4, paragraphe 2, TUE. ${ }^{86}$

Bien que la Cour de justice dans les trois arrêts mentionnés se soit exprimée à l'art. 4 du paragraphe 2 du TUE par lequels les États membres se sont protégés, dans aucun d'eux la conception de l'identité nationale n'était un argument suffisant pour la dérogation au droit de l'UE.

79 Commission européenne contre Royaume d'Espagne, C-151/12, point 28.

80 Ibid., point 37.

81 Arrêt de la Cour de justice du 17 juillet 2013, Torresi, C-58/13 et C-59/13, ECLI:EU:C:2014:2088.

82 Conclusion de l'avocat général Nils Wahl du 10 avril 2014 dans l'affaire Torresi, C-58/13 et C-59/13, ECLI:EU:C:2014: 265, point 99.

83 Ibid., point 100.

84 Torresi, C-58/13 et C-59/13, point 56.

85 Ibid., point 57.

86 Ibid., point 58. 
L'arrêt Melloni ${ }^{87}$ portait aussi sur le droit secondaire. Dans cette affaire la Cour constitutionnelle espagnole a déposé une demande de décision préjudicielle dans l'affaire où l'exécution du mandat d'arrêt européen (ci après « MAE »), concrètement l'art. 4a, paragraphe 1, de la décision-cadre relative au MAE, s'oppose au droit au procès équitable interprété par la cour constitutionnelle espagnole. D'après sa jurisprudence l'exercice de MAE délivré en vue d'exécuter un jugement rendu par défaut doit être subordonnée à la condition que la personne condamnée a le droit dans l'État membre d'émission à une nouvelle procédure. Il s'applique, en vertu de l'art. 4a, paragraphe 1 de la décision-cadre que si une telle personne a connaissance du procès prévu donne mandat à son avocat pour la défendre au procès, la remise ne pourrait pas obéir à une telle condition.

D’après l'avocat général, un État membre devrait avoir la possibilité attaquer la disposition du droit secondaire en vertu de l'art. 4, paragraphe 2 TUE au cas où une telle disposition menace son identité nationale. Ensuite, il a ajouté que dans l'affaire pendante il ne s'agit pas d'une telle situation. D'après l'avocat général la détermination de l'étendu du droit au proces équiptable et au droit à la défense dans les jugements par défaut ne peut pas toucher l'identité nationale du Royaume d'Espagne. Il en résulte surtout du fait que le contenu du droit à la défense n’est toujours pas fixé dans la jurisprudence de la Cour constitutionnelle espagnole et en plus le gouvernement espagnol lui même a mentionné que la participation de l'accusé à son procès ne relève pas de l'identité constitutionnelle du Royaume d'Espagne. Aussi, il faut faire la distinction entre le contenu de la protection des droits fondamentaux et la menace de l'identité nationale, resp. de l'identité constitutionnelle d'un État membre. Dans ce cas là, d'après l'avocat général il s'agit d'un droit fondamental protégé par la Constitution espagnole, c'est pourqoui cela signifie que l'application de l'art. 4, paragraphe 2, TUE ne doit pas être envisagée. ${ }^{88}$

La Cour de justice, sans référeces aux principe de la protection de l'identité nationale, est arrivée à ce que l'Espagne ne peut se prévaloir du droit au procès équitable au termes de la constitutions espagnole ayant pour but de dévier la décision-cadre du MAE. C'est que l'exécution du MAE n'est pas subordonné à la condition que la condamnation par défaut puisse être révisée dans l'État membre d'émission. ${ }^{89}$ D' après CJUE le MAE est compatible avec la Charte des droits fondamentaux de l'Union européenne et n'intervient nullement dans le droit au procès équitable.

Il résulte de cette argumentation que la protection nationale des droits fondamentaux ne peut pas être plus large que leur protection au niveau de l'UE puisque une interprétation d'un procès équitable prévu par la constitution espagnole n'était pas prise en considération. La Cour de justice a ainsi donné la primauté à l'uniformité du droit de l'UE et au principe de la primauté absolue résultant devant des spécificités constitutionnelles d'un État membre réflétées dans l'art. 4, paragraphe 2 du TUE.

Le droit secondaire était traité aussi dans les affaires jointes Bero Pham. ${ }^{90}$ La question était de savoir s'il était possible de retenir les immingrés des pays tiers en attente de l'éloignement dans des établissement pénitentiaire au motif qu'il n'existait pas de centres de rétention spécialisé en vertu de l'art. 16 paragraphe 1 de la directive n. 2008/115/CE. Dans la procédure, la République fédérale d'Allemagne a argumenté par l'obligation de l'UE de respecter l'identité nationale d'un État membre et elle enchaine que « le gouvernement allemand soutient qu’il serait porté atteinte à la souveraineté

87 Arrêt de la Cour de justice du 26 février 2013, Melloni, C-399/11, ECLI:EU:C:2013:107.

88 Conclusion de l'avocat général Yves Bota du 2 octobre 2012 dans l'affaire Melloni, C-399/11, ECLI:EU:C:2012:600, points $139-142$

89 Melloni, C-399/11, point 64.

90 Arrêt de la Cour de justice du 17 juillet 2014, Bero Pham, C-473/13 a C-514/13, ECLI:EU:C:2014:2095. 
administrative d'un Lander si ce dernier ne pouvait placer les migrants en attente d'éloignement dans un établissement pénitentiaire de son ressort au motif qu' il existe ailleurs sur le territoire national des centres de rétention spécialisés. ${ }^{91}$

Cependant, d'après l'avocat général « un État membre ne peut exciper de dispositions ou de pratiques nationales découlant de son organisation fédérale pour éventuellement justifier le non-respect des obligations prescrites par une directive. ${ }^{92}$ Dans le cas où un État membre a sur son territoire des centres de rétention spécialisés avec une capacité disponible suffisante, la personne interessée doit être placée dans ce centre, peut importe l'organisation fédérale de l'État. L'avocat général a soutenu cette argumentation par la condition aux États membres d'observer le principe de coopération prévu dans l'art. 4, paragraphe 3 du TUE, et s'abstenir ainsi de toutes mesures susceptibles de mettre en péril la réalisation des objectifs de l'UE. ${ }^{93}$

La Cour de justice a enchaîné à la conclusion de l'avocat général et a estimé que « l'obligation, prévue à la première phrase de l'article 16 , paragraphe 1 , de la directive 2008/115, d'effectuer la rétention en règle générale dans des centres de rétention spécialisés s'impose aux États membres en tant que tels, et non pas aux États membres en fonction de leur structure administrative ou constitutionnelle respective. ${ }^{94}$

Comme dans l'arrêt Melloni aussi dans l'arrêt Bero Pham la Cour de justice ne s'est nullement exprimée même si les États membres ont reclamé ses specificités constitutionnelles. Pour tous les arrêts mentionés dans le présent chapitre il est typique que la CJUE n’a pas une seule fois appliqué le test de proportionnalité.

\section{CONCLUSION}

Dans la jurisprudence avant Lisbonne de la Cour de justice, la notion de l'identité nationale ou bien constitutionnelle ne jouait pas un rôle important parce que pedant cette période la CJUE n'a mentionnée la notion de l'identité nationale que dans ses trois décisions. Dans tous ces arrêts la Cour n'a en plus utilisé l'identité nationale que comme un argument subsidiaire. Ainsi, la CJUE n'a probablement pas voulu restreindre la compétence du droit de l'UE dans le cas où l'obligation de respecter l'identité nationale ne la liait pas et gardait ainsi le principe de primauté absolue du droit de l'UE défini dans l'arrêt Internationale Handelsgesellschaft. ${ }^{95}$

Contrairement à la CJUE les avocats généraux comprenaient positivement la conception de l'identité nationale. Surtout l'avocat général Miguel Poiares Maduro a essayé d'apporter dans ses quelques conclusions un peu de lumière sur ce concept. En revanche, Pedro Cruz Villalón, par exemple, dans sa conclusion, sans analyse plus profondes, n'a répété qu'avec austerité l'argument d'un État membre réclamant la sauvegarde de son identité nationale. Ce qui est clé c'est surtout l'interprétation de la conception de l'identité nationale par la Cour de justice car ce sont justement ses arrêts qui sont obligatoires.

91 Conclusion de l’avocat général Yves Bota du 30 avril 2014 dans l'affaire Bero Pham, C-473/13 a C-514/13, ECLI:EU: C:2014:295, point 122 .

92 Ibid., point 143.

93 Ibid., points 144, 145.

94 Bero Pham, C-473/13 et C-514/13, point 28.

95 Arrêt de la Cour de justice du 17 décembre 1970, Internationale Handelsgesellschaft, C-11/70, ECLI:EU:C:1970:114. 
La CJUE ne commençait à se préoccuper considérablement de la conception de l'identité nationale ou bien constitutionnelle qu'après l'entrée en vigueur du Traité de Lisbonne. Ni même dans ces arrêts l'argumentation de l'art. 4 paragraphe 2 TUE ne jouait pas un rôle primaire. Même si dans ces procédures on a réglé une collision du droit européen avec les spécificités constitutionnelles des États membres et les États membres renvoyaient directement à l'art. 4 paragraphe 2 du TUE, l'argument principale de la Cour de justice a été l'argumentation résultant de sa jurisprudence constante soutenue dans certains cas par une mention de l'identité nationale.

Pour la plupart des arrêts de la Cour de justice concernant l'identité nationale il est typique que ce sont les parties de la procédure ou bien les sujets intervenants qui argumentent sur l'identité nationale ou bien constitutionnelle. Ces sujets révendiquent souvent leur identité nationale comme l'exception en droit de l'UE. Quoique la CJUE réagit à cette argumentation différement, souvent il est obligé d'adopter une position à l'identité nationale. Soit, la CJUE ne s'exprime point, soit elle assume qu'il n'est pas possible de l'appliquer dans ce cas concret, soit elle l'utilise comme un argument de soutien.

Le problème de la jurisprudence ici analysée est le fait que la CJUE n'applique pas l'art. 4 paragraphe 2 du TUE par un procédé fixe. En général, il résulte de sa jurisprudence que l'identité nationale d'un État membre peut représenter un but légitime permettant à l'État membre une dérogation au droit de l'UE. Cependant, en aucun cas l'art. 4 paragraphe 2 ne représente une dérogation automatique au droit de l'UE car dans aucun des arrêts la CJUE n'a utilisé l'identité nationale séparement comme une justification de la dérogation au droit de l'UE. La Cour de justice relie en général l'identité nationale à sa jurisprudence constante et à l'application du principe de proportionalité. Néamoins dans certains cas on n'arrive jamais à parvenir à l'application du test de proportionalité.

La CJUE enchaîne avec ses décisions précédentes où elle a accepté différents principes du droit constitutionnel interne d'un État membre sans référence à l'identité nationale comme la justification de la restriction des droits fondamentaux. De cette catégorie relève aussi par exemple la liberté de réunion et d'expression, ${ }^{96}$ la dignité humaine ${ }^{97}$ ou bien la pluralité des médias. ${ }^{98}$ Dans ces décisions aussi que par exemple dans Sayn-Wittgenstein, Runevič-Vardyn et Digibet Albers, la CJUE a donné aux autorités nationales une certaine liberté pour la considération. Ce qui est différent c'est que l'objectif identique, c'est-à-dire le respect de certaines spécificités constitutionnelles des États membres, dans les affaires précédentes étaient atteint même sans faire références à l'identité nationale des États membres. ${ }^{99}$

En réalité, en application de l'art. 4 paragraphe 2 du TUE, la CJUE n'a pas crée de nouvelle approche aux restrictions au droit de l'UE mais elle n'a que fait classé l'art. 4 paragraphe 2 du TUE dans son arsenal des arguments qui sont conformes à son actuelle pratique décisionnelle. ${ }^{100} \mathrm{La}$ CJUE a ansi « neutralisé » la conception de la sauvegarde de l'identité nationale et par sa elle lui a ôté son contenu potentiellement explosif. ${ }^{101}$

Le résultat de chaque procédure devant la CJUE où se présente l'argumentation par l'identité nationale dépend des circonstances concrètes de l'affaire. De la jurisprudence actuelle de la CJUE ne résulte

96 Arrêt de la Cour de justice du 12 juin 2003, Schmidberger, C-112/00, ECLI:EU:C:2003:333.

97 Omega, C-36/02.

98 Arrêt de la Cour de justice du 26 juin 1997, Familiapress, C-368/95, ECLI:EU:C:1997:325.

99 CLAES: National Identity..., s. 133.

100 TONIATTI, Roberto. Sovereignty Lost, Constitutional Identity Regained. In: ARNAIZ, Alejandro Saiz, LLIVINA, Carina Alcoberro (ed). National Constitutional Identity and European Integration. Cambridge: Intersentia, 2013, p. 71.

101 CLAES: National Identity..., p. 134 
pas un mécanisme constant qui généralement pourrait être appliqué dans le cas d'une collision du droit de l'UE avec des spécificités résultants des Constitutions des États membres. Au contraire, il s'agit d'un équilibre classique des intérêts divergents faisant partie de la jurisprudence constante de la CJUE.

En définissant la conception de l'identité nationale ou bien constitutionnelle il faut prendre en considération le fait que la CJUE ne peut pas interpréter le droit national des États membres et c'est pourquoi pour elle, reste clé la coopération avec les acteurs nationaux qui peuvent remplir l'argument de l'identité nationale par un contenu correspondant pour chaque État membre. On ne peut pas comprendre le principe de l'art. 4 paragraphe 2 du TUE par l'analyse de la jurisprudence seulement d'un acteur car le respect de l'identité national est toujours le résultat de dialogues des acteurs européens et nationaux et donc il a toujours deux faces : nationale et internationale. ${ }^{102}$ Les arguments des institutions nationales peuvent résulter aussi de la jurisprudence constante des juridictions nationales constitutionnelles. Il reste ainsi à la Cour de justice d'évaluer leur pertinence du point de vue du droit de l'UE. Cette mutuelle coopération résulte entre autre du principe de la coopération loyale entre les acteurs européens et nationaux conformément à l'art. 4 paragraphe 3 de TUE.

Le problème est que la CJUE non seulement s'exprime très peu dans ces décisions sur l'identité mais elle transforme aussi dans sa jurisprudence constante les prétentions qui en résultent. C'est une démarche complétement contraire à celui par lequel les juridictions constitutionnelles nationales interprétent l'identité nationale ou bien constitutionnelle. ${ }^{103}$

L'art. 4 paragraphe 2 du TUE est ainsi par la jurispridence constante de la Cour de justice retransformé de la collision du droit européen avec les principes résultant des constitutions nationales dans la question se rapportant à l'incorporation des intérêts divergents et des principes justifiant la dérogation du droit de l'UE qui cependant résultent du droit de l'UE même ou bien de la jurisprudence de la Cour de justice. ${ }^{104}$

À la question posée tout au début on peut répondre que la CJUE par sa jurisprudence n'a pas crée de l'art. 4 paragraphe 2 TUE une conception autonome qui pourrait lui seul justifier la dérogation au droit de l'UE, mais il ne fait que l'attacher à sa jurisprudence constante.

\section{RÉFÉRENCES}

\section{Ressources scientifiques:}

Arnaiz, A.S. and Llivina, C.A. 2013. 'Why Constitutional Identity Suddenly Matters: A Tale of Brave States, a Mighty Union and the Decline of Sovereignty'. In Arnaiz, A.S. and Llivina, C.A. (eds.) National Constitutional Identity and European Integration. Cambridge: Intersentia.

Besselink, L.F.M. 2010. 'National and Constitutional Identity before and after Lisbon'. Utrecht Law Review (6) 3:36-49.

Claes, M. (2013). 'National Identity: Trump Card or Up for Negotiation?'. In Arnaiz, A.S. and Llivina, C.A. (eds.) National Constitutional Identity and European Integration. Cambridge: Intersentia.

Gisbert, R. B. 2013. 'National Constitutional Identity in European Constitutionalism: Revisiting the Tale of the Emperor's New Clothes in Spain'. In Arnaiz, A.S. and Llivina, C.A. (eds.) National Constitutional Identity and European Integration. Cambridge: Intersentia.

102 GISBERT, Rafael Bustos. National Constitutional Identity in European Constitutionalism: Revisiting the Tale of the Emperor's New Clothes in Spain. In: ARNAIZ, Alejandro Saiz, LLIVINA, Carina Alcoberro (ed). National Constitutional Identity and European Integration. Cambridge: Intersentia, 2013, p. 76.

103 Par exemple la décision de de la Cout fédéral constitutionnll du 30 juin 2009, 2 BvE 2/08 Lissabon-Urteil.

104 CLAES: National Identity..., p. 130. 
Grewe, C. 2013. 'Methods of Identification of National Constitutional Identity'. In Arnaiz, A.S. and Llivina, C.A. (eds.) National Constitutional Identity and European Integration. Cambridge: Intersentia.

Kumm, M., Ferreres Comella, V. 2005. 'The Primacy Clause of the Constitutional Treaty and the Future of Constitutional Conflict in the European Union'. International Journal of Constitutional Law (3) 2-3: 473-492.

Martí, J.L. 2013. 'Two Different Ideas of Constitutional Identity: Identity of the Constitution v. Identity of the People'. In Arnaiz, A.S. and Llivina, C.A. (eds.) National Constitutional Identity and European Integration. Cambridge: Intersentia.

Martinico, G. 'What Lies behind Article 4(2) TEU'. In Arnaiz, A.S. and Llivina, C.A. (eds.) National Constitutional Identity and European Integration. Cambridge: Intersentia.

Toniatti, R. 2013. 'Sovereignty Lost, Constitutional Identity Regained'. In Arnaiz, A.S. and Llivina, C.A. (eds.) National Constitutional Identity and European Integration. Cambridge: Intersentia.

Von Bogdandy, A., Schill, S. 2011. 'Overcoming Absolute Primacy: Respect for National Identity under the Lisbon Treaty'. Common Law Market Review (48) 5: 1417-1453.

Zbíral, R. 2014. 'Koncept národní identity jako nový prvek ve vztahu vnitrostátního a unijního práva: Poznatky z teorie a praxe' '. Právník (Juriste), (153), 2: 112-133.

\section{Ressources judiciaires:}

Arrêt de la Cour de justice du 1 mars 2012, O’Brien, C-393/10, ECLI:EU:C:2012:110.

Arrêt de la Cour de justice du 12 juin 2003, Schmidberger, C-112/00, ECLI:EU:C:2003:333.

Arrêt de la Cour de justice du 12 juin 2014, Digibet Albers, C-156/13, ECLI:EU:C:2014:1756.

Arrêt de la Cour de justice du 12 mai 2011, Runevič-Vardyn, C-391/09, ECLI:EU:C:2011:291.

Arrêt de la Cour de justice du 14 octobre 2004, Omega, C-36/02, ECLI:EU:C:2004:614.

Arrêt de la Cour de justice du 16 avril 2013, Anton Las, C-202/11, ECLI:EU:C:2013:239.

Arrêt de la Cour de justice du 16 décembre 2008, Michaniki, C - 213/07, ECLI:EU:C:2008:731.

Arrêt de la Cour de justice du 17 décembre 1970, Internationale Handelsgesellschaft, C-11/70, ECLI:EU:C:1970:114.

Arrêt de la Cour de justice du 17 juillet 2013, Torresi, C-58/13 et C-59/13, ECLI:EU:C:2014:2088.

Arrêt de la Cour de justice du 17 juillet 2014, Bero Pham, C-473/13 a C-514/13, ECLI:EU:C:2014:2095.

Arrêt de la Cour de justice du 2 juillet 1996, Commission des Communautés européennes contre Grand-Duché de Luxembourg, C-473/93, ECLI:EU:C:1996:263.

Arrêt de la Cour de justice du 22 décembre 2010, Ilonka Sayn-Wittgenstein, C-208/09, ECLI:EU:C:2010:806.

Arrêt de la Cour de justice du 24 mai 2011, Commission européenne contre le Grand-duché de Luxembourg, C-51/08, ECLI:EU:C:2011:336.

Arrêt de la Cour de justice du 24 octobre 2013, Commission européenne contre Royaume d'Espagne, C-151/12, ECLI:EU:C:2013:690.

Arrêt de la Cour de justice du 26 février 2013, Melloni, C-399/11, ECLI:EU:C:2013:107.

Arrêt de la Cour de justice du 26 juin 1997, Familiapress, C-368/95, ECLI:EU:C:1997:325.

Arrêt de la Cour de justice du 28 novembre 1989, Groener, C-379/87, ECLI:EU:C:1989:599.

Conclusion de l'avocat général Dámas Ruiz-Jarabo Colomer du 25 juin 2009 dans l'affaire Umweltanwalt von Kärnten, C-205/08, ECLI:EU:C:2009:397

Conclusion de l'avocat général Juliane Kokott du 21 juin 2011 dans l'affaire République italienne contre Commission européenne, C 566/10 P, ECLI:EU:C:2012:368.

Conclusion de l'avocat général Juliane Kokott du 30 mai 2013 dans l'affiare Commission européenne contre Royaume d'Espagne, C-151/12, ECLI:EU:C:2013:354.

Conclusion de l'avocat général Léger du 5 mars 1996 dans l'affaire Commission contre Grand-Duché de Luxembourg, C-473/93, ECLI:EU:C:1996:80. 
Conclusion de l'avocat général Miguel Poiares Maduro du 16 décembre 2004 dans l'affaire Royaume d'Espagne contre Eurojust, C-160/03, ECLI:EU:C:2004:817.

Conclusion de l'avocat général Miguel Poiares Maduro du 20 septembre 2005 dans l'affaire Marrosu Vassallo, C - 53/04, ECLI:EU:C:2005:569.

Conclusion de l'avocat général Miguel Poiares Maduro du 30 septembre 2009 dans l'affiare Rottmann, C-135/08, ECLI:EU:C:2009:588.

Conclusion de l'avocat général Miguel Poiares Maduro du 8 octobre 2008 dans l'affaire Michaniki, C - 213/07, ECLI: EU:C:2008:544.

Conclusion de l'avocat général Niilo Jääskinen du 16 décembre 2010 dans l'affaire Runevič-Vardyn, C-391/09, ECLI: EU:C:2010:784.

Conclusion de l'avocat général Nils Wahl du 10 avril 2014 dans l'affaire Torresi, C-58/13 et C-59/13, ECLI:EU:C: 2014:265.

Conclusion de l'avocat général Pedro Cruz Villalón du 14 septembre 2010 dans l'affaire Commission européenne contre le Grand-Duché de Luxembourg, C-51/08, ECLI:EU:C:2010:525.

Conclusion de l'avocat général Yves Bot du 2 octobre 2012 dans l'affaire Melloni, C-399/11, ECLI:EU:C:2012:600.

Conclusion de l'avocat général Yves Bot du 30 avril 2014 dans l'affaire Bero Pham, C-473/13 a C-514/13, ECLI: EU:C:2014:295.

Conclustion de l'avocat général Verica Trstenjak du 4 juin 2008 dans l'affaire Coditel Brabant, C-324/07, ECLI:EU: C:2008:317.

Conclution de l'avocat général Niilo Jääskinen du 12 juillet 2012 dans l'affaire Anton Las, C-202/11, ECLI:EU:C:2012: 456.

Décision de de la Cout fédéral constitutionnll du 30 juin 2009, 2 BvE 2/08 Lissabon-Urteil.

Décision de la Cour constitutionnelle fédérale du 12 octobre 1993, BverfGE 89, 155 Maastricht-Urteil.

\section{Contact information:}

JUDr. Ondrej Hamulák, Ph.D

ondrej.hamulak@upol.cz

Faculty of Law, Palacký University Olomouc

Mgr. David Kopal

kopaldvd@gmail.com

Faculty of Law, Palacký University Olomouc

tr. 17. listopadu 8

Olomouc 77111

Czech Republic

prof. Tanel Kerikmäe, Ph.D.

tanel.kerikmae@ttu.ee

Tallinn Law School, Tallinn University of Technology

Akadeemia tee 3

Tallinn 12618

Estonia 